\title{
Load Frequency control(LFC) of a Multiarea Restructured Hybrid Powersystem on Multi objective SSA
}

\author{
VSR Pavan Kumar Neeli, USalma
}

\begin{abstract}
The aimof the paper tune the paramters of the load frequency controller using a latest and novel algorithm named as Salp sarm of algorithm with multiobjective approach. The test system choosen is a Two area interconnected hybrid power system under deregulated-environment integrated with Distributd genertion $(D G)$ resource.The DG systems consists of Windturbine generator(WTG), SolarPV systems, Diesels engines generators(DEG), Fuelcells with Aqua electrolyzers and Energy storages like Batteries energy storage systems(BESS). To minimise the frequency of oscillations, Secondarycontroller opted was an optimal Fuzzy PID plus double integral controller (FPID-II). The effectiveness of proposed controller is determined with the comparison of nominal PI, PID and Two degree freedom PID (TDOFPID) controller. Furthermore the dynamic responses of SSA tuned FPID-II controller are been compared with other optimization techniques. The results depit the superiority of the proposed controller in suppressing the deviations of frequency.
\end{abstract}

Keywords : Hybrid power system, Salp swarm algorithm, Fuzzy PID plus double integral controller, MATLAB/Simulink.

\section{INTRODUCTION}

Modern new power generating system looking toward deregulationprocess that invites new challenging issues in its maintanance operation and control action. The potential stand alone generation units are capablesof producing bulkpower which categories theneed of itsown territories andalso interconneted controlareas follows thederegulatin processin which, the verticals powers generating systems divided in separateunits as GENCOs, TRANSCOs, DISCOs \& ISO [15]. One deregulatd powersystem consists inter-connected different powers generating models suchas thermal-gas, nuclear etcs. involves large dynamic respondig change of loaddisturbance. Thesedynamic ineach area frequency's and tielines powers exchanges regulate, else whichotherwise lead to deviation in frequencies andisland operations mayinitiate a major and severeblackout of inter-connected powersystems [16-17]. Henc necessaryto maintain regulated and stabilized the frequency's and tielines powers for loadchange disturbance.

With-the increases of power demandsthere is was protocal needto explore the power generation models in inter-connected powersystems and to invoke generations by

Revised Manuscript Received on December 13, 2019.

V.S.R.Pavan Kumar.Neeli*, Assistant Profesor, Dept. of EEE, Sir.C.R.Reddy college of Engineering, Eluru, Andhra Pradesh, INDIA. E-mail: pavanscholar123@gmail.com

Dr.U.Salma, Associate Professor, , Dept.of EEE, GITAM(Deemed to be University), Visakhapatam, Andhra Pradesh, INDIA. presenting newpotentialgenerating units. Withthe evident of potentials generation models suchas nuclear plants with rating over $5 \mathrm{MW}$ and to maintain the change of load rapidly, a major sources to provides the required auxillary based sevices in the deregulatedpower system [18]. Inthis papers the Distributed generation resource been implementeed along oneofthe GENCO's supply the power regulating action in deregulatedpowersystem.

Themain contributions of present works were sumarised below

$>$ Todesign modelof Two area inter-connected hybrid powersystem under deregulated environment integrated with Distributedgeneration (DG) resources.

$>$ To get the parameters of different controllers using various optimization techniques.

$>$ Compare the dynamic performances of all controllers and to judge which controller gives effective and satisfactory results.

Deepak kumar and Ajit kumar [1] has established a fuzzy PIDcontroller for aSinglearea hybrid powersystem in which the controller parameters are tuned with Moth flame optimization. Similarly pandey et.al [2] has presented a control scheme of linear matrix inequalities for the single area hybrid system along with a two area hybrid power system. The LMI approch is developed with the help of Geneticalgorithm \& Particlesswarm optimization algorithms. Sarada prasanna Behera et.al [3-4] demostrates the loadfrequencycontrol(LFC) problem for a Twoarea inter-connected Hybrid powersystems with two types ofcontrollers like Hybrid PIDF controller and a TID controller, Differential evolution(DE) is used for parameter extraction of the respective controllers. Raju et.al [5] presents the frequency control problem of three area interconnected Hybrid power system with secondary controller considered as Two degree of Freedom controller, Symbiotic organisms search (SOS) techniquesis applied to obtain the parameters of the controllers. Yogendra arya [6] has proposed a Fuzzy PID with filter plusdouble integrall controller with out scaling factors for AGC of twoarea electric power system.

\section{DEREGULATED POWER SYSTEM MODEL}

In the present paper work two area inter-connected thermal power system integrated with Distributed generation (DG) resource under deregulated environment model is considered for the investigation. 
The deregulated power system has as many as GENCOs and DISCOs. DISCO and GENCO will have a contract for transactions of powers. If DISCO in any of the controlarea has a contracts with a GENCOin the samearea it is noted as "Pool-co" transaction and if a DISCO have a contracts with a GENCO in the other control areas, then that type of transaction is called as "Bilateral" transaction.

The concepts ofthe DISCOParticipationMatrix(DPM) is usedto calculate the contract between a DISCO's and GENCO. The DPM was a matrix in which the no of rowsequal tothe noof GENCO and thenoof columnsequal to the noof DISCO of thesystems. The matrix entry's are treated as contract participation factors. Equation 1 gives the DPM for the proposed power system[19].

$$
D P M=\left[\begin{array}{llll}
c p f_{11} & c p f_{12} & c p f_{13} & c p f_{14} \\
c p f_{21} & c p f_{22} & c p f_{23} & c p f_{24} \\
c p f_{31} & c p f_{32} & c p f_{33} & c p f_{34} \\
c p f_{41} & c p f_{42} & c p f_{43} & c p f_{44}
\end{array}\right] \text { with } \quad \sum c p f_{i j}=1
$$

Where, ${ } p f_{i j}=\frac{\text { DemandofDisco }^{\prime} j^{\prime} \text { fromGenco' } i^{\prime}}{\text { TotalDemandofDisco' } j^{\prime}}$

The block diagrams of the proposed powersystem model isshown at Figur1, andits first order transferfunction model isdescribedin Figur3. Inthis modelsthe expressins foractuals \& scheduleds steadystate powerflow onthe tielines [20] weregivenas

$$
\begin{aligned}
& \Delta \text { Ptie }_{12, \text { schedule }}=\sum_{i=1}^{2} \sum_{j=3}^{4} c p f_{i j} \Delta P_{L j}-\sum_{i=3}^{4} \sum_{j=1}^{2} c p f_{i j} \Delta P_{L j} \\
& \Delta \text { Ptie }_{12, \text { schedule }}=\left(c p f_{13}+c p f_{23}\right) \Delta P_{L 3}+\left(c p f_{14}+c p f_{24}\right) \Delta P_{L 4} \\
& -\left(c p f_{31}+c p f_{41}\right) \Delta P_{L 1}-\left(c p f_{32}+c p f_{42}\right) \Delta P_{L 2} \\
& \Delta \text { Ptie }_{12, \text { actual }}=\frac{2 \Pi T_{12}}{s}\left(\Delta f_{1}-\Delta f_{2}\right)
\end{aligned}
$$

The tielines powererror ( $\triangle \mathrm{P}$ tie 12error) isdefinedby

$$
\Delta \text { Ptie }_{12, \text { errors }}=\Delta \text { Ptie }_{12, \text { actuals }}-\Delta \text { Ptie }_{12, \text { scheduld }}
$$

The area control error(ACE) in an deregulated powersystem in both areas are defined as

$$
\begin{gathered}
A C E_{1}=B_{1} \Delta f_{1}+\Delta \text { Ptie }_{12, \text { error }} \\
A C E 2=B_{2} \Delta f_{2}+\alpha_{12} \Delta \text { Ptie }_{12, \text { error }}
\end{gathered}
$$

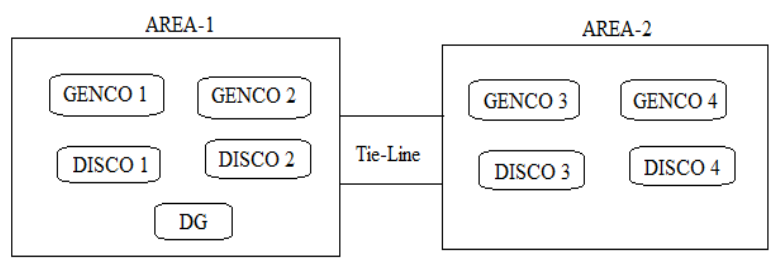

Fig 1: Twoarea Interconnected Thermal power system integrated with DG in area-1 under Deregulated Environment

The parameters that contribute Area Control Error to the participating GENCO are represented asACE's Participation Factors (APF's). The total sum of the APF's in a respective area should be equals as unity.

$$
\begin{aligned}
& a p f_{1}+a p f_{2}=1 \\
& a p f_{3}+a p f_{4}=1
\end{aligned}
$$

From Figure 3, the respective contracts are listed as $\Delta \mathrm{P}_{1}, \Delta \mathrm{P}_{2}$,

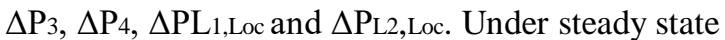

condition, contracts of DISCOs with GENCOs are shown in equations 9-14,

$\Delta \mathrm{P}_{\mathrm{L} 1 \_ \text {Loc }}=\Delta \mathrm{P}_{\mathrm{L} 1}+\Delta \mathrm{P}_{\mathrm{L} 2}$

$\Delta \mathrm{P}_{\mathrm{L} 2 \_ \text {Loc }}=\Delta \mathrm{P}_{\mathrm{L} 3}+\Delta \mathrm{P}_{\mathrm{L} 4}$

$\Delta \mathrm{P}_{1}=\operatorname{cpf}_{11} \Delta \mathrm{P}_{\mathrm{L} 1}+\operatorname{cpf}_{12} \Delta \mathrm{P}_{\mathrm{L} 2}+\operatorname{cpf}_{13} \Delta \mathrm{P}_{\mathrm{L} 3}+\operatorname{cpf}_{14} \Delta \mathrm{P}_{\mathrm{L} 4}$ $\Delta \mathrm{P}_{2}=\operatorname{cpf}_{21} \quad \Delta \mathrm{P}_{\mathrm{L} 1}+\mathrm{cpf}_{22} \quad \Delta \mathrm{P}_{\mathrm{L} 2}+\mathrm{cpf}_{23} \quad \Delta \mathrm{P}_{\mathrm{L} 3}+\mathrm{cpf}_{24} \Delta \mathrm{P}_{\mathrm{L} 4}$ (12)

$\Delta \mathrm{P}_{3}=\operatorname{cpf}_{31} \quad \Delta \mathrm{P}_{\mathrm{L} 1}+\operatorname{cpf}_{32} \quad \Delta \mathrm{P}_{\mathrm{L} 2}+\operatorname{cpf}_{33} \Delta \mathrm{P}_{\mathrm{L} 3}+\operatorname{cpf}_{34} \Delta \mathrm{P}_{\mathrm{L} 4}$ (13)

$$
\Delta \mathrm{P}_{4}=\operatorname{cpf}_{41} \Delta \mathrm{P}_{\mathrm{L} 1}+\operatorname{cpf}_{42} \Delta \mathrm{P}_{\mathrm{L} 2}+\mathrm{cpf}_{43} \Delta \mathrm{P}_{\mathrm{L} 3}+\operatorname{cpf}_{44} \Delta \mathrm{P}_{\mathrm{L} 4}
$$

\section{DISTRIBUTED GENERATION (DG) RESOURCES MODEL}

For simulating of the largescalesystems, simplifiedmodels suchastransfer functions model areto bebuilted. Hences powergenerating unitsare modelled asfirstorder form of tranfer funtion model. Therefore the totalpower obtained was the combinationsof powersfrom the thermalsunits and powers fromthe DGsystem resorces [12]. Theoutputpower ofDistributed Generation system isgiven

$\Delta P_{D G}=P_{W g}+P_{P v}+P_{D g}+P_{F c}-P_{A e} \pm P_{B s s}$

For smallsignal stability purpose, the generation unitslike Windpower genertors, SolarPV, Fuelcells \& electrolyzers and Diesels generators canbe modelledby the firstordered transferfunction withparameter gainsand timeconstant [14]. Thelinearized models ofvarious generating systemsare representedbelow

$$
\begin{gathered}
H_{g W}(s)=\frac{K_{W g}}{1+T_{W g} s} \\
H_{P V}(s)=\frac{K_{P V}}{1+T_{P V} s} \\
H_{F c}(s)=\frac{K_{F c}}{1+T_{F c} s} \\
H_{D g}(s)=\frac{K_{D g}}{1+T_{D g} s} \\
H_{A e}(s)=\frac{K_{A e}}{1+T_{A e} s} \\
H_{B s s}(s)=\frac{K_{B s s}}{1+T_{B s s} s}
\end{gathered}
$$

\section{A. Fuzzy PID plus double Integral (FPID-II) Controller}

General layover of FPID-II controller shown in the fig.1. The perfromance of this controllers depends upon five dfferent controller parameters includng the scalng factors. The parameters values are been tuned with the help of SSA optimization technique. Apart fromcontroller parameterrs the fuzzy logic controller (FLC)useserror and derivativeoferror asinput signlas to getthe best performance ofthe FPID-II controller. 


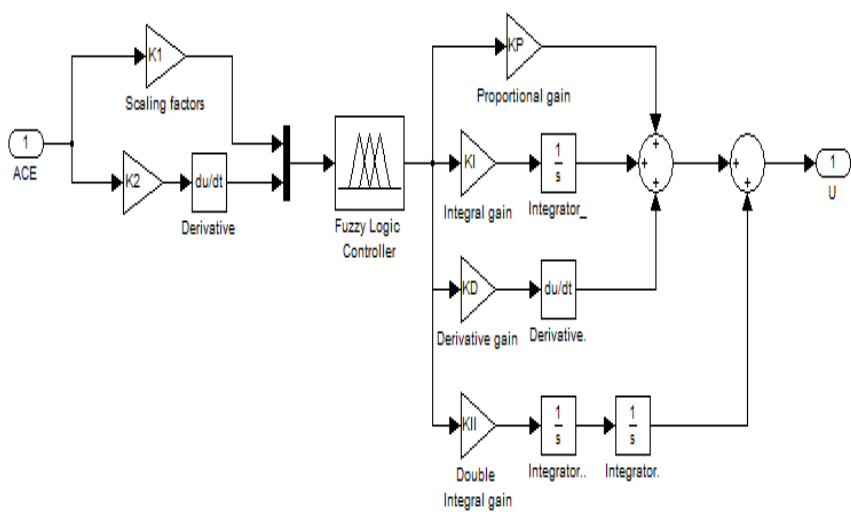

Fig 2: Structure of FPID-II controller

The control signal of the poposed controller given below

$$
U=K p+\frac{K i}{s}+K d . s+\frac{K i i}{s^{2}}
$$

Different membership fuections such as traingular,

\begin{tabular}{|c|c|c|c|}
\cline { 2 - 4 } $\mathrm{e}$ & $\mathrm{N}$ & $\mathrm{Z}$ & $\mathrm{P}$ \\
\hline $\mathrm{N}$ & $\mathrm{N}$ & $\mathrm{N}$ & $\mathrm{Z}$ \\
\hline $\mathrm{Z}$ & $\mathrm{N}$ & $\mathrm{Z}$ & $\mathrm{P}$ \\
\hline $\mathrm{P}$ & $\mathrm{Z}$ & $\mathrm{P}$ & $\mathrm{P}$ \\
\hline
\end{tabular}

Table1 Rule base for error, derivative of error and FLC output

\section{OBJECTIVE FUNCTION}

The present work was carried out with an Integral of Square errror $\{$ ISE $\}$ is chosen as a desired objective functions for the tuning of proposed FPID-II controller. In this paperfor the Hybrid powersystem considered two objective and three objective functions [13] are employed for the tuning process which are listed below.

Two objective Function

$$
\begin{aligned}
& J_{1}=\min \int_{0}^{T}\left(\Delta f_{1}^{2}+\Delta f_{2}^{2}+\Delta P_{T i e 12}^{2}\right) d t \\
& \mathbf{J} 2=\text { Minimum Overshoot }\{(\Delta \mathrm{f} 1)+(\Delta \mathrm{f} 2)+(\Delta \text { Ptie } 12)\}
\end{aligned}
$$

Where ' $\mathrm{J}$ ' minimized subjected to

$$
\left.\begin{array}{lc}
K_{1}^{\min } \leq K_{1} \leq K_{1}^{\max } & K_{2}^{\min } \leq K_{2} \leq K_{2}^{\max } \\
K_{P}^{\min } \leq K_{P} \leq K_{P}^{\max } & K_{I}^{\min } \leq K_{I} \leq K_{I}^{\max } \\
K_{D}^{\min } \leq K_{D} \leq K_{D}^{\max } & K_{I I}^{\min } \leq K_{I I} \leq K_{I I}^{\max }
\end{array}\right\}
$$

\section{OVERVIEW OF SALP SWARM ALGORITHM}

SalpSwarm Algorithm (SSA) is an novel Swarm-intelligence algorithm [7] developed by Prof.Mirjalili. SSA was a populations base method which illustrates mimicking behaviours of Salp Swarm and their interactions. Thegroupof the Salp calledsalpchainsmathematically divided into twogroups heads salp was a leader andother arefollowers. Tillnow, thebehaviour of salpsswarms was notwell considered. Therefore the researcherscholars considers the behaviors of it thei movement inseeking thefood.

\section{Stepfollowed inthe SSA}

1. Parameters-initializatin:The algorithm starts by initializng parameter suchas size in population $N$, and noof iterationt, \& maximumiteration $\max _{i t e r}$.

2. Initial-Populations: The generates initial populations $x_{i} i=\{1 \ldots . . n\}$ random inbetween ranges of $[u, l]$ where $u, l$ are upperlower boundarysrespectivey.

3. Individual-Evaluations: Each individual in the populations were evaluated by calculating the objective functions values and the overall best solution was assigned for Function.

4. Explorations \& exploitations: To maintain balance between Exploration \& exploitation of algorithm, we need to update value of parameter $c_{l}$ mentioned in the equation

$$
c_{1}=2 e^{-\left(\frac{4 l}{L}\right)^{2}}
$$

Where $l$ the presentiterations and $L$ isthe max noof Iterations.

5. Positions updation of thesolutions: The positions of the leader solutions and other followers solution are updated as

$$
x_{j}^{1}=\left\{\begin{array}{l}
F_{j}+c_{1}\left(\left(u b_{j}-l b_{j}\right) c_{2}+l b_{j}\right. \\
F_{j}-c_{1}\left(\left(u b_{j}-l b_{j}\right) c_{2}+l b_{j}\right.
\end{array}\right\} \begin{aligned}
& \text { forc }_{3} \geq 0 \\
& \text { forc }_{3}<0
\end{aligned}
$$

Where the $x_{j}^{l}$ is the leader positions in $j^{\text {th }}$ dimensions and $u b_{j} \& l b_{j}$ are the maximum and min boundary for $j^{\text {th }}$ dimensions and $F_{j}$ is the food source positions.

And $x_{j}^{i}=\frac{1}{2}\left(x_{j}^{i}+x_{j}^{i-1}\right)$

Where $i \geq 2 ; x_{j}^{i} \quad$ denote the positions of $i^{t h}$ followers Salp in the $j^{\text {th }}$ dimensions.

6. Boundaryviolations: If solutions violate the ranges of the searchspace engine during updateflow, it returns back the ranges of the problem.

7. Terminationcriteria: The no of iteration $t$ was increased rapidly untill it reache to max iterations $\max _{i t e r}$ so the algorithm gets terminated searching processand produce the overall the bestsolutions got until so far. 

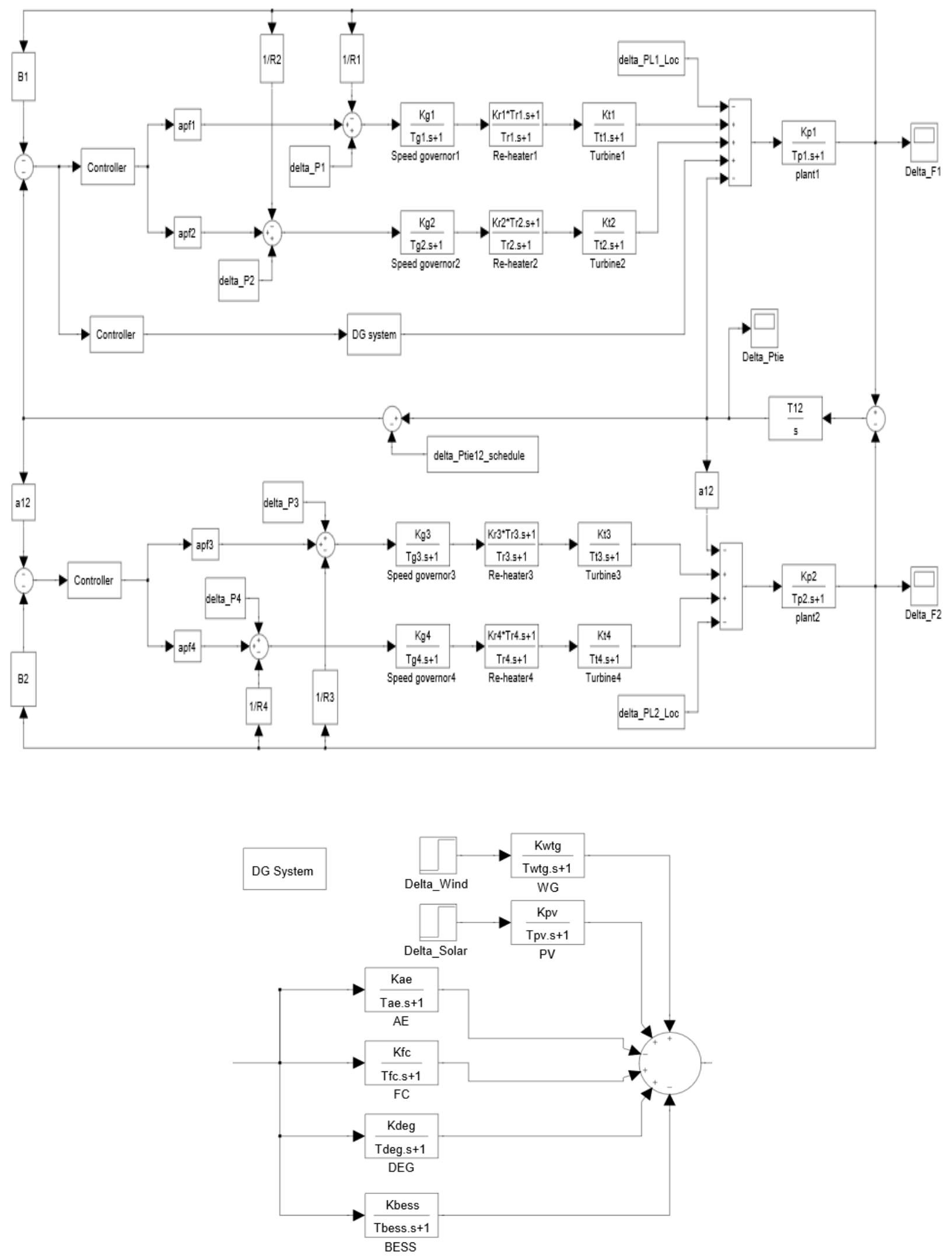

Figs 3: Twoarea Inter-connected Thermal powersystems integrated with DG inarea-1 under Restructured Environment 


\section{RESUltS AND DiscuSSIONS}

The Dynaamic behaviour of the choosen powersystems is analyzed with two different scenarios.Simulation were conducted at an Intel, Core i-3, 4 GB RAM computers in the MATLAB (R2010a) software environment. The windpower and solarpower variations appliedtothe powerssystems are consideredas $\Delta \mathrm{P}_{\mathrm{wtg}}=0.5 \mathrm{p}$.uand $\Delta \mathrm{P}_{\mathrm{pv}}=0.18 \mathrm{p}$.u.

Initially the comparisons of dynamic responses of PI, PID, TDOFPID \& FPID-II controller is carried out with the SSA technique. The results depicts that the FPID-II controller performs better controlling action compared to PI, PID and TDOFPID. Furthermore the comparison of dynamic responses of SSA, GOA, ALO, DA and PSO [8-11] are carried out with FPID-II controller for different loading conditions.

\section{Case 1: Pool-co based Transaction}

If a DISCO's in one controlarea has a contracts with a GENCO in the same areas it is noted as "Pool-co" transaction.

In Poolco based contract the change in load has been considered in area1 only. Letthisload demands forDISCO1 andDISCO 2 tobe 0.1 puMW foreach ofthem. Therefore, the demands of DISCOs in (p.u.MW) is

$$
\begin{gathered}
\Delta \mathrm{P}_{\mathrm{L} 1}=\Delta \mathrm{P}_{\mathrm{L} 2}=0.1 \text { and } \\
\Delta \mathrm{P}_{\mathrm{L} 3}=\Delta \mathrm{P}_{\mathrm{L} 4}=0
\end{gathered}
$$

$D P M=\left[\begin{array}{llll}c p f_{11} & c p f_{12} & c p f_{13} & c p f_{14} \\ c p f_{21} & c p f_{22} & c p f_{23} & c p f_{24} \\ c p f_{31} & c p f_{32} & c p f_{33} & c p f_{34} \\ c p f_{41} & c p f_{42} & c p f_{43} & c p f_{44}\end{array}\right]=\left[\begin{array}{cccc}0.5 & 0.5 & 0 & 0 \\ 0.5 & 0.5 & 0 & 0 \\ 0 & 0 & 0 & 0 \\ 0 & 0 & 0 & 0\end{array}\right]$

The elements that contribute ACE to the participating GENCOs are represented as ACEParticipationFactors(APF) givenas $\operatorname{apf}_{1}=\operatorname{apf}_{2}=\operatorname{apf}_{3}=\operatorname{apf}_{4}=0.5$.

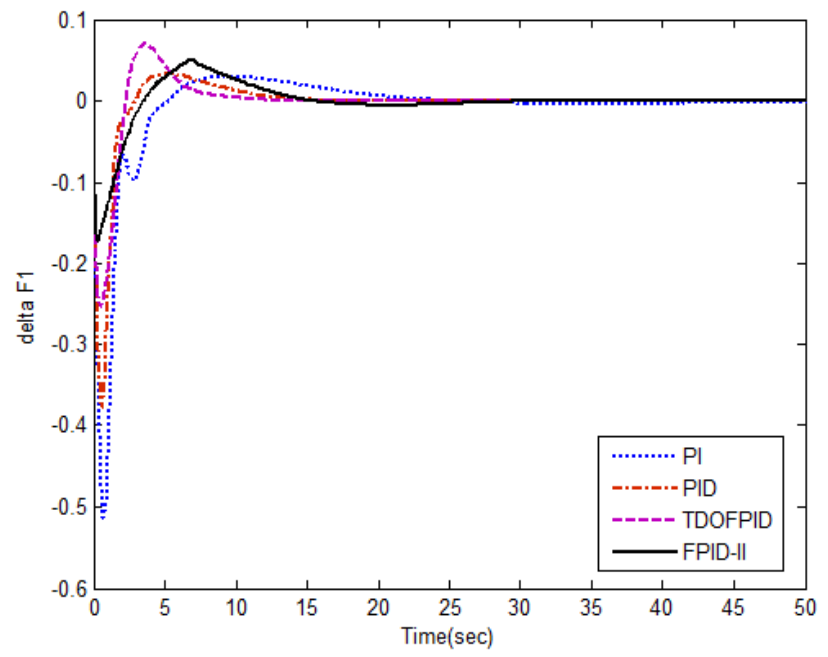

Figs 4: Deviationof frequencyinarea-1

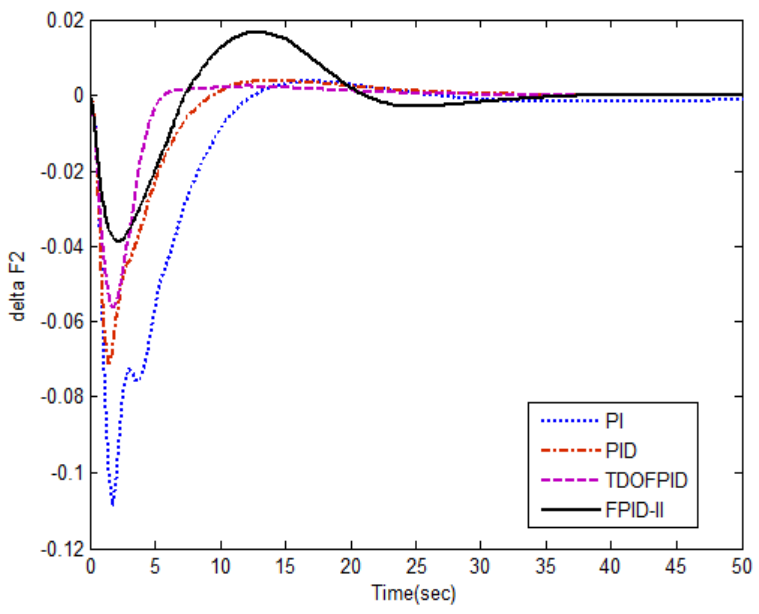

Figs 5: Deviationof frequencyinarea-2

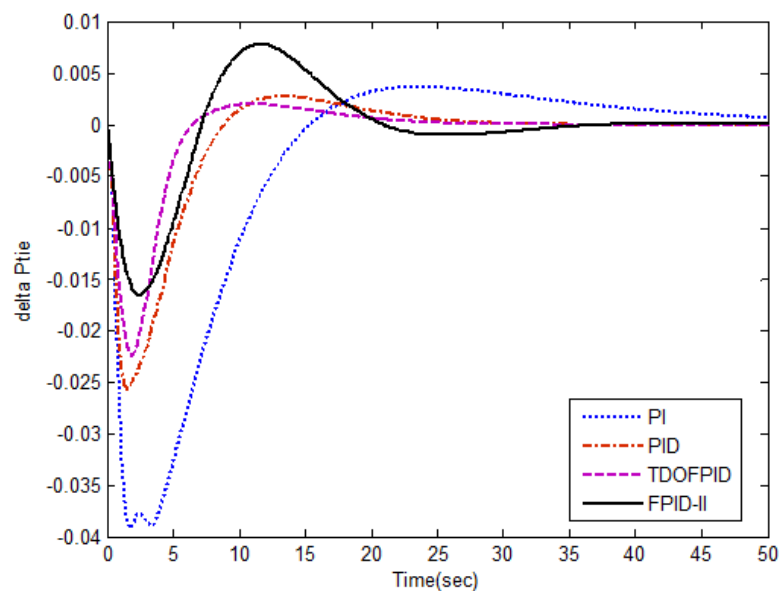

Figs 6: DeviationofTielinespower

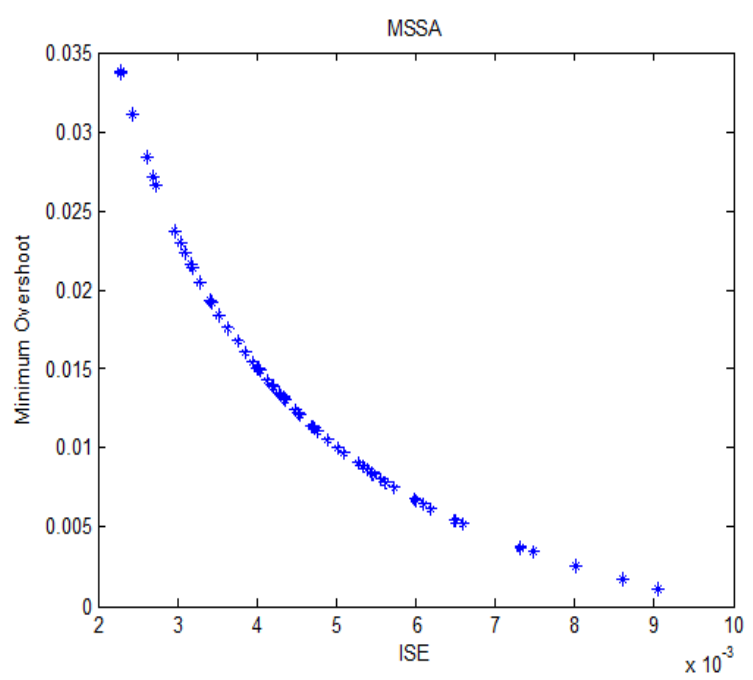

Figs 7: Optimalresponse for Twoobjective

\section{Case 2: Bilateral Transaction}

In case 2 all the DISCO's are in contracts with allthe GENCO's of their respective area for trasaction of power. Let this load demand for all the DISCO's be 0.1puMW. Thereforethe demands of DISCOs in (puMW) is

$$
\Delta \mathrm{P}_{\mathrm{L} 1}=\Delta \mathrm{P}_{\mathrm{L} 2}=\Delta \mathrm{P}_{\mathrm{L} 3}=\Delta \mathrm{P}_{\mathrm{L} 4}=0.1
$$


DISCO participation matrix (DPM) is given as

$D P M=\left[\begin{array}{llll}c p f_{11} & c p f_{12} & c p f_{13} & c p f_{14} \\ c p f_{21} & c p f_{22} & c p f_{23} & c p f_{24} \\ c p f_{31} & c p f_{32} & c p f_{33} & c p f_{34} \\ c p f_{41} & c p f_{42} & c p f_{43} & c p f_{44}\end{array}\right]=\left[\begin{array}{cccc}0.5 & 0.25 & 0 & 0.3 \\ 0.2 & 0.25 & 0 & 0 \\ 0 & 0.25 & 1 & 0.7 \\ 0.3 & 0.25 & 0 & 0\end{array}\right]$

The elements that contributed ACE's to the participating GENCO are represented as

ACE'sParticipationFactors(APF's) given

apf1 $=0.75 \mathrm{apf}_{2}=0.25 \mathrm{apf}_{3}=0.5 \mathrm{apf}_{4}=0.5$.

The DISCO have contract with all GENCOs irrespective of areas as this is a Bilateral transaction, then

$$
\Delta P_{\text {tie12 }}^{\text {schedule }}=-0.05 \text { p.u. } M W
$$

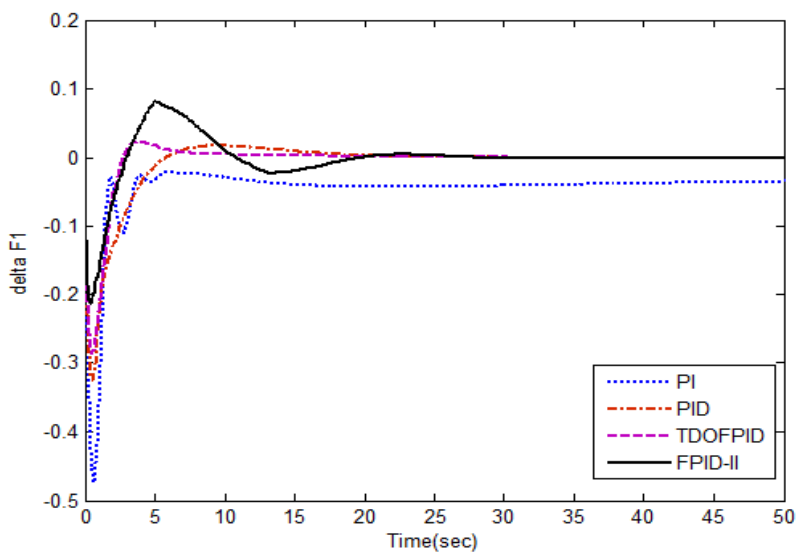

Figs 8: Deviationoffrequencyinarea-1

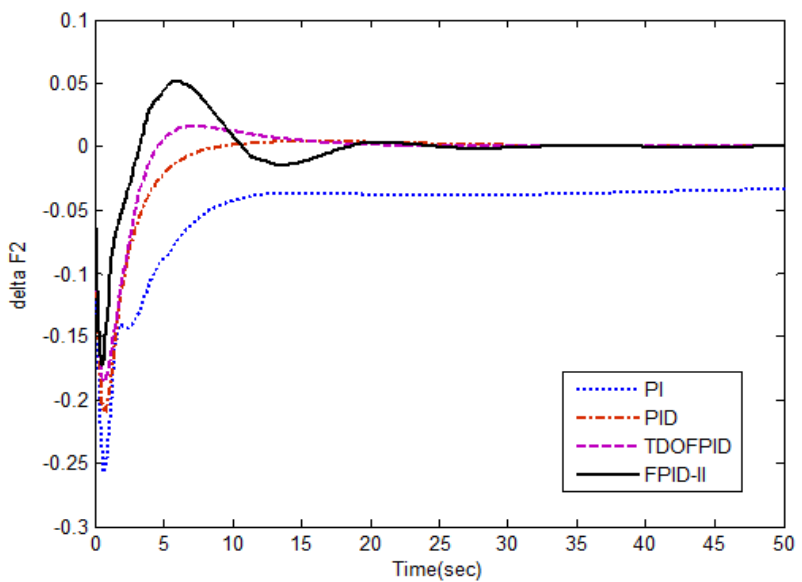

Figs 9: Deviationoffrequencyinarea-2

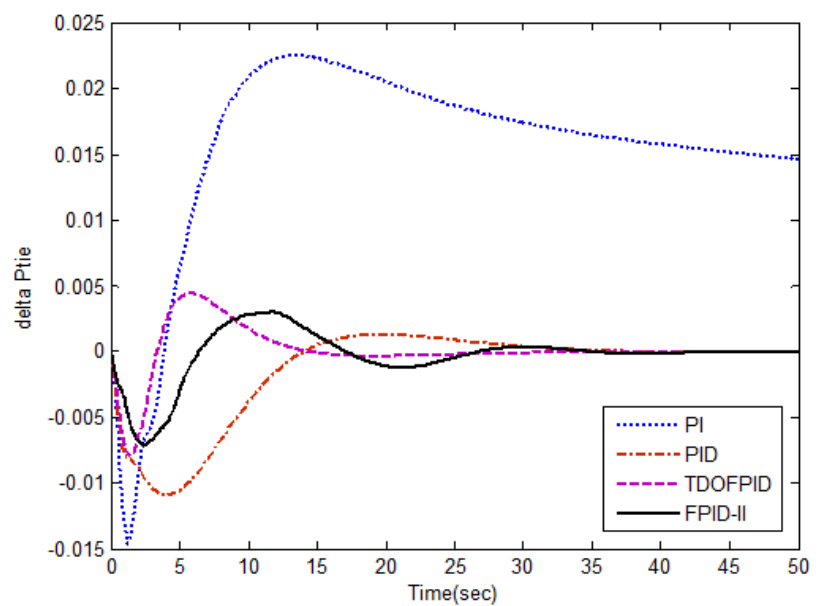

Figs 10: DeviationofTielinepower

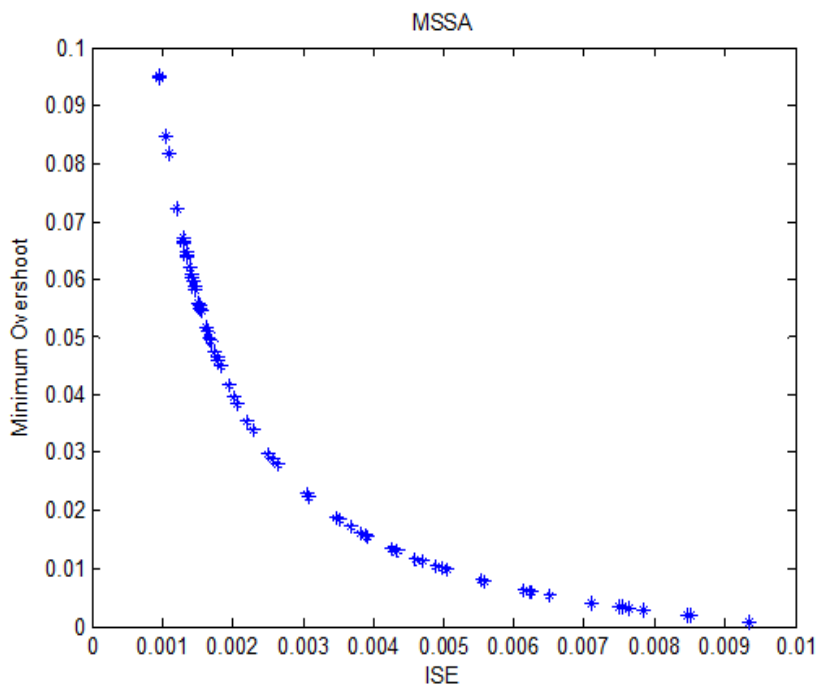

Figs 11: Optimal response for Two objective

From the above results, it is clearly evident that for two objective functions, the proposed controller (FPID-II) establishes a better and sophisticated results when compared with other controllers. The respective objective functions values of allcontroller are listed in the table. 2

Case 3: Comparison of SSA responses with different optimization techniques

In order to extract effectiveness ofSSA technique the respective SSA responses arebeing compared withother technniques such an Antlion optimization (ALO), Grasshopper optimization algoithm (GOA), Dragonfly algorithm (DA), Particleswarrm optimization(PSO).

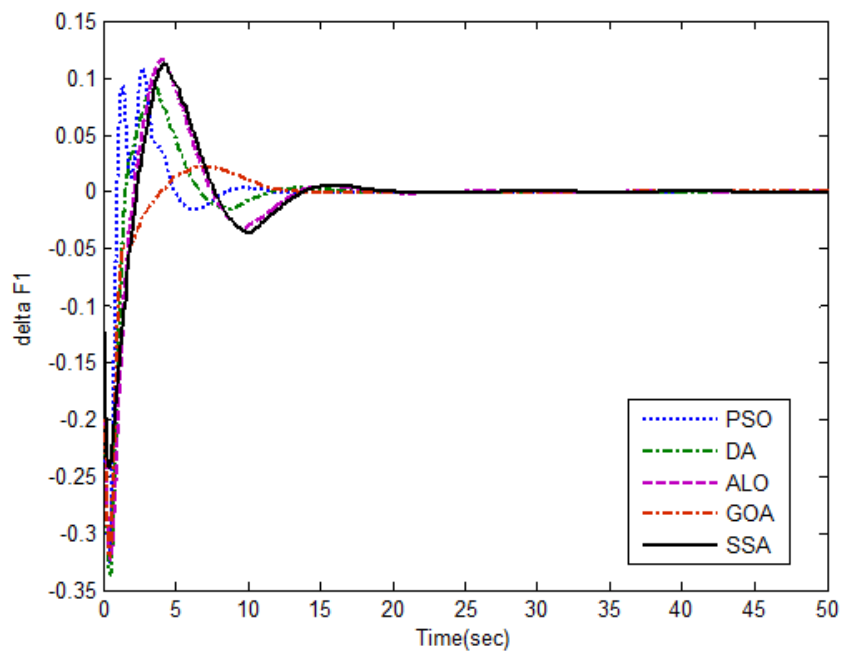

Fig 20: Deviation in frequency in area-1 


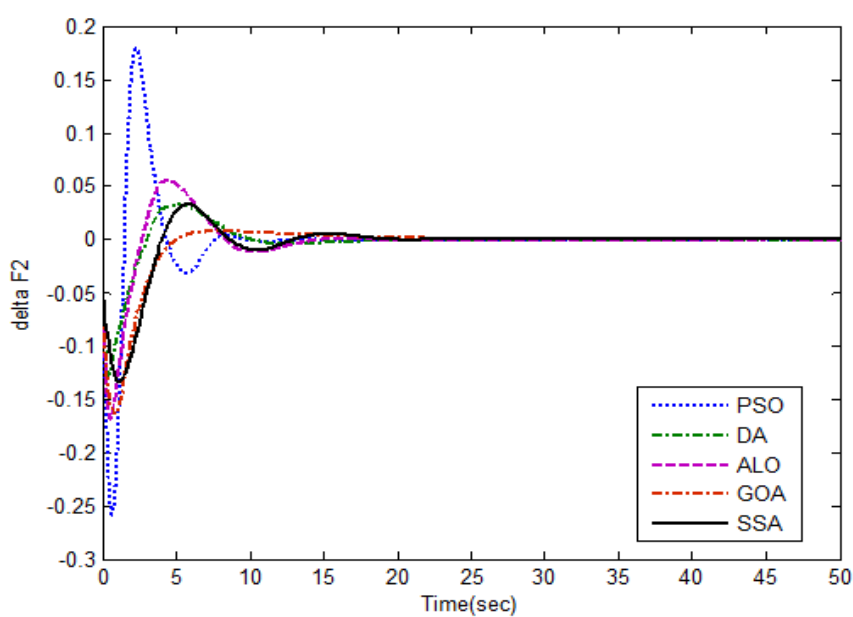

Fig 21: Deviation in frequency in area-2

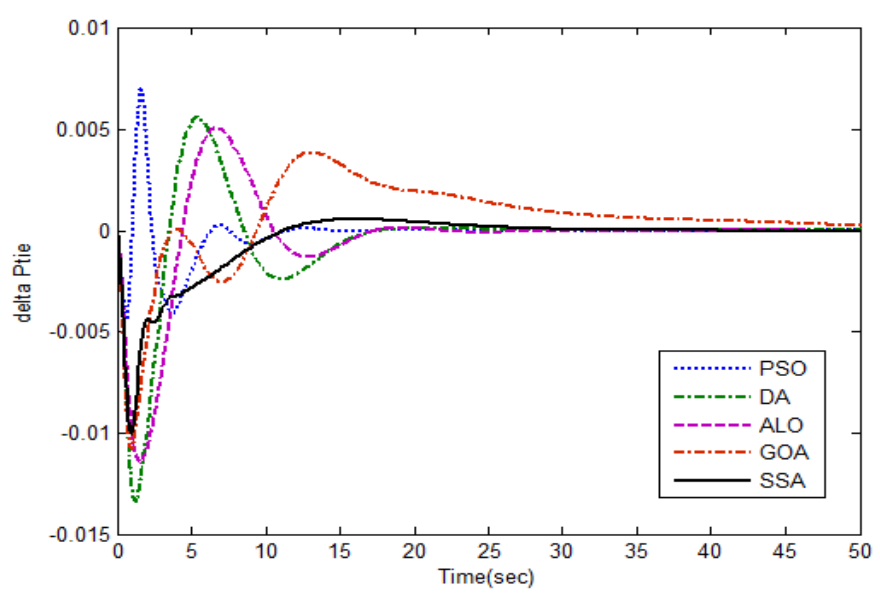

Fig 22: Deviation in Tie-line power
After critical inspection from above results, reveals that the proposed FPID-II controller exhibits the robustness and renowned for both the considered cases.

\section{CONCLUSION}

Salp swarm algorithm (SSA) based Fuzzy PID plusdouble integral (FPID-II)controller is considered forthe AGC problem of the restructured Hybrid powersystem such as Twoarea inter-connected thermal power system integrated with Distributed generation (DG) resources under deregu-lated environment. Performances of different controllers are compared with SSA technique. The parameter of PI, PID, TDOFPID andFPID-IIcontrollers are obtained by employing SSA algorithm with multi objective approach and their respective performances are been compared for both pool-co and bilateral transactions.A critical studiesof the obtaineddynamics response revealsthat FPID-II controlleris superiorkeeping inview ofminimising the frequency oscillations than other controllers.

Moreover, the responses obtained from SSA technique are compared with other optimization techniques and it was observed that the proposed SSA tuned FPID-II controller is a robust and promising controller, which ableto hold the demands and generations under aderegulated environment for different transaction.

\begin{tabular}{|c|c|c|c|c|}
\hline \multirow[t]{3}{*}{ Controllers } & \multicolumn{4}{|c|}{$\begin{array}{c}\text { Twoarea Inter-connected Thermal powersystems integrated with DG in area-1 under } \\
\text { restructured Environment }\end{array}$} \\
\hline & \multicolumn{2}{|c|}{$\begin{array}{r}\text { Poolco } \\
\end{array}$} & \multicolumn{2}{|c|}{ Bilateral } \\
\hline & $\mathrm{J}_{1}$ & $\mathrm{~J}_{2}$ & $\mathrm{~J}_{1}$ & $\mathrm{~J}_{2}$ \\
\hline PI & 0.0030 & 0.0495 & 0.0036 & 0.3283 \\
\hline PID & 0.0025 & 0.0478 & 0.0028 & 0.2640 \\
\hline TDOFPID & 0.0017 & 0.0236 & 0.0022 & 0.1788 \\
\hline FPID-II & 0.0012 & 0.0140 & 0.0017 & 0.0497 \\
\hline
\end{tabular}

Table 2: Comparison of objective function values of considered controllers

\section{REFERENCES}

[1] Lal Deepak Kumar and Ajit Kumar Barisal "Loa frequency control of AC micro grid inter-connected thermal powers systems" IOP Conferences Series: Materials Sciences and Engineering Vol225No1IOPPublishing2017

[2]PandeShashiKat"Frequecy regulating in hybrids powers system using particles swarms optimization and linear matrix in equalities base robust cotrollersdesig" InternationalJournalfElectricalsPowers \&EnergySyste mss 2014p887to900

[3]BeheraSaradPrasanna"HybridspowerssystemFrequecyregulatinusing HybridsPIDFbaserobustcontrllerdesignandDiffeentialEvoltion(DE) algorthms2018TehnologiesforSmartCitEnergySecuritandPowersICSE SP IEEE2018

[4]BeheraSaradPrasanna"HybridspowerssystemFrequecyregulatinusing TIDbaserobustcontrllerdesignandDiffeentialEvoltion(DE)
algorthms2018TehnologiesforSmartCitEnergySecuritandPowersICSE SP IEEE2018

[5]SaikiLalitChandraandNidulSinha"Loadfrequencycontrolofmultiareashybri dspowerssystemsusingssymbiotiorganimsseachoptimzedtwodegreoffreed ocontrolerInternatinalJournalofRenewablEnergesearchIJRER2017,16 63 to1674

[6]AryaYogendra" Automticgeneratincontroloftwoareaelectricalspower ssystemsviaoptimalsfuzzyclassiclcontroller"JournaloftheFranklinInstitu t2018pp2662to2688

[7]MirjaliliSeyedali"Salps Swarms Algrithm:Abioinspired optimzerfor engineringdesign problemss" Advance inEnginering Software 2017 pp163to191

\section{PublishedBy:}

BlueEyesIntelligenceEngineering 
Load Frequency control(LFC) of a Multiarea Restructured Hybrid Powersystem on Multi objective SSA

[8]MirjaliliSeyedeZahra"Grasshoperoptimzationalgorthmformulti-objetive optimzation problems"AppliedIntellgence 2018pp805to820

[9]MirjalilSeyedalPradeepJangrandShahzadSaremi"Multis-objectivesantliono ptimizers:multis-objectivesoptimzationalgorithmsforsolvingenginering problemss"AppliedIntellgenc2017pp79to95

[10]MirjalilSeyedali"Dragonflysalgorithms:newmeta-heursticoptimzation techniquesforsolvingssingle-objectivediscreteandmulti-objetiveproblems " NeuralComputngan Application2016pp1053to1073

[11]KennedyandEberhart"Particlesswarmoptimzation(PSO)" ProcIEEE InternatinalConferencesonNeuralNetworkssAustralia1995pp1942to1 948

[12]DasDulalAKRoyand NSinha"GAbasedfrequencyscontrollersforsolar thermals-diesels-windd hybrid senergysgenerationenergystorage systems"InternationalJournalofElectricalsPowers\&EnergysSystem 201 2pp262to279

[13]SeshadriAravind"Multisobjectivesoptimizationsusingevolutionary algorithmss MOEA2006pp1to20.

[14] ShankarGandMukherjee"Loadsfrequencyscontrolsoffautonomouss hybridspowerssystems byy quasii-oppostional harmonyssearch algorthm"InternationaJouna ofElectricals Powers\&EnergysSystemss 2016 pp715to734

[15] LaLoLei.Powerssystemsrestucturingandderegultiontrading performnce andinformatioontechnologysNewYork Wiley2001

[16] DondVaibhaMAPaiandIanAHiskens"Simulationsandoptimizastionsin anAGCsystemsafterdereguation"IEEE transactions on power syste 2001 pp481to489

[17] Shayeghi HAShayanfarandAJalili"LFCsdesignsofdereguulatedpowers systemswithTCPSusingsPSO"InternationnalJourrnalElectrricaland ElectrronicEngineering2009pp632to640

[18]KakimotNaotandKazuhirBaba"Performaance off gasturbiine bassed plantduriingfrrequencydroops"TransactioononPowers SystemsIEEE 2003 pp1110to1115

[19] LakshmiDFathimandRanganathMuthu"Simulatioon oofTwoArea DerregulatedPowerr0Systemmusing ParticlesSwarms Optimizaations "Interrnationa l Jourrnalon Electrrical Engineerring \& Inforrmatics 2016

[20] Ibraheeem"Optimallautomaticegenerrationcontrrolofinterrconnected powerrsystemmwith aasynchronous tieliness under derregulated ennvironments "Electrric PowerrCompponentt andSysstems 2012 pp1208to1228

\section{AUTHORS PROFILE}

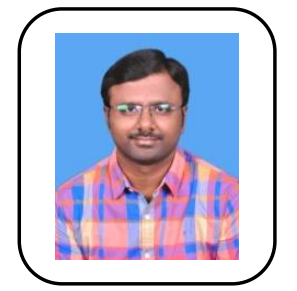

VSR Pavan Kumar Neeli is pursuing Ph.D in departmentof ElectricalElectronicscommunication Enginering GITAM Institute of Technology, GITAM(Deemedtobe University,Visakhapatnam, A.P India under the guidance of Dr.U.Salma. He received his B.Techdegree in the deparment of Electrical and ElectronicsEngineering from JNTUniversityKakinada India in 2009 and M.E degreein the Department of ElectricalEngineering from AndhraUniversity, Visakhapatnam in 2012. He is working as an Assistant Profesor in Sir.C.R.Reddy college of Engineering, Eluru since 2012. His research interests include Load frequency control, Controller deisgn using computational Techniques.

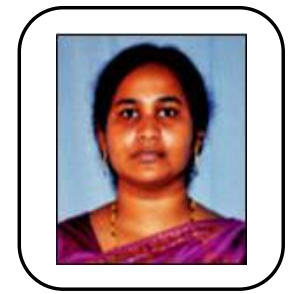

Dr.U.Salma is currently working as an Associate Professorin department of Electrical Electronics andcommunication Enginering GITAM Instituteof Technology GITAM (Deemed to be University), Visakhapatnam, A.P India. She received her B. Tech and $M$. Tech Degres in Electrical and Electronics Engineering from JNTUniversity Hyderabad, Indiain 2002 and 2006 respectively. She received her Ph.D in Electrical and Electronics Engineeringfrom JNTUniversity, Kakinada, India in 2016. Her research interests includeApplications of Model Order Reduction Techniques to Power Systems. 